Mathematical Research Letters 5, 213-226 (1998)

\title{
A DICHOTOMY FOR FORCING NOTIONS
}

\author{
JINDŘICH ZAPLETAL
}

Abstract. Let $B$ be Baumgartner's poset for adding a closed unbounded subset of $\omega_{1}$ with finite conditions. Under the Proper Forcing Axiom, a forcing notion $P$ of size $\aleph_{1}$ is nowhere c.c.c. if and only if $B$ completely embeds into $P$.

\section{Introduction}

Among the partial orderings of size $\leq \aleph_{1}$ the following four stand out:

- adding one Cohen real or $C_{\aleph_{0}}$, with the associated complete algebra denoted by $\mathbb{C}_{\aleph_{0}}$

- adding $\aleph_{1}$ Cohen reals or $C_{\aleph_{1}}$, with completion $\mathbb{C}_{\aleph_{1}}$

- adding a closed unbounded subset of $\omega_{1}$ by finite conditions denoted by $B$ with the completion $\mathbb{B}$-see $[\mathrm{B}]$

- the collapse of $\aleph_{1}$ to $\aleph_{0}$ denoted by $D$ with the completion $\mathbb{D}$.

It has been shown that modulo forcing equivalence these are the only simply definable posets of size $\aleph_{1}$ [Z2], and external characterizations of the complete Boolean algebras in question were obtained $[\mathrm{K}, \mathrm{Z1}, \mathrm{J}]$. [SZ] proves a basic dichotomy theorem:

Theorem 1. Assume the Proper Forcing Axiom holds. Then every separative poset $P$ of size $\aleph_{1}$

- either has a countable somewhere dense subset

- or $\mathbb{C}_{\aleph_{1}}$ can be completely embedded into $R O(P)$, in short, $P$ adds $\aleph_{1}$ Cohen reals.

Here, a subset of $P$ is somewhere dense if there is a condition $p \in P$ under which it is dense. Theorem 1 shows that $\mathbb{C}_{\aleph_{1}}$ is in a certain precise sense the simplest complete Boolean algebra of uniform density $\aleph_{1}$. In this paper we show

Theorem 2. Assume the Proper Forcing Axiom holds. Then every poset $P$ of size $\aleph_{1}$

- either is somewhere c.c.c.

- or $\mathbb{B}$ can be completely embedded into $R O(P)$, in short, $P$ adds a club with finite conditions.

Received May 1, 1997.

1991 Mathematics Subject Classification. 03E40, 06E10.

The author is partially supported by grant GA ČR 201/97/0216. 
Recall that a poset $P$ is somewhere c.c.c. if there is a condition $p \in P$ such that every antichain below $p$ is countable. Thus Theorem 2 shows that $\mathbb{B}$ is the simplest among all complete Boolean algebras of density $\aleph_{1}$ in which c.c.c. fails everywhere. By an inspection of the proofs we obtain

Theorem 3. Assume the Proper Forcing Axiom holds. Then for every partial ordering $P$ of size $\aleph_{1}$ and every stationary set $S \subset \omega_{1}$

- either some condition in $P$ forces the stationarity of $S$ to be preserved

- or $B_{\omega_{1} \backslash S}$ can be completely embedded into P.

Here the forcing $B_{X}$ for $X \subset \omega_{1}$ is the variation of $B$ adding a closed unbounded subset of $X$, and the above result says that under PFA this is the simplest such a poset of size $\aleph_{1}$.

Consider the quasiorder of complete embeddability $\lessdot$ between complete Boolean algebras and say that algebras $\mathbb{A}_{0}, \mathbb{A}_{1}$ are equivalent if $\mathbb{A}_{0} \lessdot \mathbb{A}_{1} \lessdot \mathbb{A}_{0}$. Thus under PFA $\mathbb{C}_{\aleph_{1}}$ is the smallest among all algebras of uniform density $\aleph_{1}$ and $\mathbb{B}$ is the smallest among all nowhere c.c.c. algebras of density $\aleph_{1}$. There is one important difference between $\mathbb{C}_{\aleph_{1}}$ and $\mathbb{B}$ which should be pointed out. In ZFC it can be proved $[\mathrm{K}]$ that $\mathbb{C}_{\aleph_{1}} \lessdot \mathbb{A} \lessdot \mathbb{C}_{\aleph_{1}}$ implies $\mathbb{A}$ isomorphic to $\mathbb{C}_{\aleph_{1}}$. Therefore the equivalence class of $\mathbb{C}_{\aleph_{1}}$ contains only its isomorphs and in ZFC it is minimal among algebras of uniform density $\aleph_{1}$ in the considered quasiordering. This is not the case for $\mathbb{B}$. In ZFC there are examples of complete algebras $\mathbb{A}$ with $\mathbb{B} \lessdot \mathbb{A} \lessdot \mathbb{B}$ and $\mathbb{A}$ not isomorphic to $\mathbb{B}$, as well as examples of algebras strictly between $\mathbb{C}_{\aleph_{1}}$ and $\mathbb{B}$-see $[\mathrm{Z} 1]$. Under the Continuum Hypothesis there is a nowhere c.c.c. algebra $\mathbb{A}$ with $\mathbb{B} \nless \mathbb{A} \lessdot \mathbb{B}$ and so one cannot prove in ZFC even the minimality of $\mathbb{B}$ among the nowhere c.c.c. algebras of density $\aleph_{1}$. Another ZFC fact further clarifying the structure of the quasiorder $\lessdot$ has been demonstrated in [Z1]: given stationary sets $S, T \subset \omega_{1}$ then $\mathbb{B}_{S} \lessdot \mathbb{B}_{T}$ just in case $T \subset S$ modulo the nonstationary ideal and the two algebras are isomorphic if and only if the sets $S, T$ are equal modulo the ideal.

One has to resort to some additional axioms in order to obtain above Theorems 1,2,3 since for instance in the context of the Continuum Hypothesis their conclusions fail badly. However, there is no need for the large cardinal strength of the Proper Forcing Axiom; indeed, the conclusions can be shown equiconsistent with ZFC.

Our notation follows the set-theoretic standard as set forth in $[\mathrm{J}] .0$ is treated as a limit of limit ordinals, $[\alpha, \beta)$ denotes the half open interval of ordinals $\gamma$ with $\alpha \leq \gamma<\beta$. In forcing we use the western conventions: smaller condition is the more informative one. Given partial orders $P, R$ the expression $P \lessdot R$ says that there is a complete subalgebra of the completion $R O(R)$ of the poset $R$ which is isomorphic to $R O(P)$. The Cohen poset $C_{\aleph_{1}}$ is construed as consisting of finite functions from $\omega_{1}$ to 2 ordered by reverse inclusion. The letter $B$ stands for Baumgartner's forcing defined in an equivalent form in [B]: a typical condition in $B$ is a pair $\langle a, b\rangle$ such that $a$ is a finite set of countable ordinals containing 0 and $b$ is a finite collection of bounded clopen intervals of countable 
ordinals disjoint from $a$. The ordering is given by $\left\langle a_{0}, b_{0}\right\rangle \geq\left\langle a_{1}, b_{1}\right\rangle$ if $a_{0} \subset a_{1}$ and $\bigcup b_{0} \subset \bigcup b_{1}$. The $B$-generic club is defined as $\bigcup\{a:\langle a, 0\rangle$ is in the generic filter $\}$-note that we always include 0 in this club for notational reasons. There are some variations of this poset we will use below. Given limit ordinals $\alpha \in \beta$ let $B_{\alpha \beta}$ be the set of pairs $\langle a, b\rangle$ with $a$ a finite subset of the interval $[\alpha, \beta)$ containing $\alpha$ and $b$ a finite collection of clopen bounded subintervals of $[\alpha, \beta)$ which are disjoint from $a$. The $B_{\alpha \beta}$ generic club is defined in the same way as the $B$-generic club; it always includes the ordinal $\alpha$. If $X$ is a set of ordinals the forcings $B_{X}, B_{[\alpha, \beta) \cap X}$ are defined similarly with the proviso that $a \subset X$. The expression $\prod_{\alpha, \beta \in \omega_{1}} B_{\alpha \beta}$ stands for the finite support product of the posets $B_{\alpha \beta}, \alpha \in \beta \in \omega_{1}$ limit ordinals.

\section{Preliminary considerations}

It is obvious that if $\mathbb{B}$ completely embeds into another Boolean algebra then that algebra must be nowhere c.c.c. since $\mathbb{B}$ is. The nontrivial part of Theorem 2 therefore lies in proving under the Proper Forcing Axiom that whenever $P$ is a nowhere c.c.c. poset of size $\aleph_{1}$ then $\mathbb{B}$ can be completely embedded into $R O(P)$. Fix such a forcing $P$ and suppose for now that $P$ is separative, preserves $\aleph_{1}$ and its universe is $\omega_{1}$. We wish to produce -in ZFC-a proper forcing which introduces a $P$-name for a $B$-generic club.

A rough description of the name runs as follows. First, we shall embed the finite support product $\prod_{\alpha, \beta \in \omega_{1}} B_{\alpha \beta}$ into $P$, with a corresponding $P$-name $\dot{H}$ for a generic filter on that poset. Note that $\prod_{\alpha, \beta \in \omega_{1}} B_{\alpha \beta}$ is really isomorphic to $C_{\aleph_{1}}$, so this is possible using the work of [SZ]. The $B$-generic club $\dot{E} \subset \check{\omega}_{1}$ will then be pieced together from the closed unbounded subsets $\dot{E}_{\alpha \beta}$ of the intervals $[\alpha, \beta)$ introduced by the $\alpha \beta$-component of the filter $\dot{H}$. For that purpose we shall add a $P$-name $\dot{C}$ for a closed unbounded subset of $\omega_{1}$ and set $\dot{E}=\bigcup\left\{\dot{E}_{\alpha \beta}: \alpha \in \beta\right.$ are successive points of $\dot{C}$ \}. A delicate interplay between the names $\dot{H}$ and $\dot{C}$ will be required in order to conclude that $\dot{E}$ is indeed a $P$-name for a $B$-generic club.

Now onto the combinatorial heart of the argument. By a proper notion of forcing we shall add

(A) a dense set $D \subset P$ of finite character, that is, for every $p \in P$ the set $\{q \in D: q \geq p\}$ is finite

(B) a $P$-name $\dot{C}$ for a club of $\omega_{1}$ consisting of limits of limit ordinals and containing 0

(C) a trace function $\operatorname{tr}: D \rightarrow\left[\omega_{1}\right]^{<\aleph_{0}}$ such that $p \Vdash$ " $\operatorname{tr}(p) \subset \dot{C}$ " and given a condition $p \in D$, a finite set $u \subset D$ of conditions $\nsupseteq p$ and a finite set $b$ of clopen bounded intervals of countable ordinals which are disjoint from $\operatorname{tr}(p)$ there is a condition $q \leq p$ such that $q$ is incompatible with every element of $u$ and it forces $\bigcup b$ to be disjoint from $\dot{C}$.

Note that $(\mathrm{A}, \mathrm{B}, \mathrm{C})$ are interdependent: that is, an existence of a trace function as in (C) implies other fine combinatorial properties of $D$ and $\dot{C}$ than those 
mentioned in (A,B). Intuitively, the only affirmative information a condition $p \in D$ carries about the generic filter and the set $\dot{C}$ is hidden in the finite sets $\{q \in D: q \geq p\}$ and $\operatorname{tr}(p)$. The above objects can be viewed as an upgrade of the strongly unbounded sets of [T2, Section 8] and the almost avoidable sets of [SZ]; the set $D \subset P$ is almost avoidable by (C). In some sense, $(\mathrm{A}, \mathrm{B}, \mathrm{C})$ are properties combinatorially optimal for our purpose. One can prove that if $B$ can be embedded into a poset $P$ of size $\aleph_{1}$ then there are $D, \dot{C}, t r$ as above. Observe that the set $\operatorname{tr}(p)$ contains 0 and consists of limits of limit ordinals since it can be forced to be a part of $\dot{C}$.

In this section it will be proved that achieving $(\mathrm{A}, \mathrm{B}, \mathrm{C})$ above is almost as good as embedding $B$ into $P$. More exactly, suppose $D, \dot{C}, \operatorname{tr}$ satisfy the requirements (A,B,C). Then $C_{\aleph_{1}} \Vdash \check{B} \lessdot \check{P}$. Force over $V$ functions $f, g: D \rightarrow 2$ with finite approximations. Obviously, $V[f][g]$ is a $C_{\aleph_{1}}$-generic extension of the ground model $V$ and it will be enough to find a complete embedding of $B$ into $P$ in $V[f][g]$.

In $V[f]$, let $D_{0}=\{p \in D: f(p)=0\}$ and $D_{1}=\{p \in D: f(p)=1\}$. So the sets $D_{0}, D_{1}$ constitute a partition of $D$ into two dense parts. As in [SZ, Claim 4]

(1) in $V[f]$ there is a family $\left\{A_{\alpha}: \alpha \in \omega_{1}\right\}$ of pairwise disjoint maximal antichains included in the set $D_{0}$.

To see this, first partition the set $D_{0}$ into $\omega_{1}$ pieces in a sufficiently generic manner. Then these pieces will be again dense in $P$ and the maximal antichain $A_{\alpha} \subset P$ can be taken as a subset of the $\alpha$-th piece. The elementary bookkeping arguments are left to the reader.

Now in $V[f][g]$ define a name $\dot{K}$ for a function from $\omega_{1}$ into 2 by $P \Vdash \dot{K}(\check{\alpha})=$ $\check{g}(p)$ where $p$ is the unique element of $\check{A}_{\alpha}$ in the generic filter. We shall show that $\dot{K}$ is a $P$-name for a $C_{\aleph_{1}}$-generic function and compute the associated projection function. Let $k: D \rightarrow C_{\aleph_{1}}$ be defined by $\operatorname{dom}(k(p))=\left\{\alpha \in \omega_{1}: \exists q \in A_{\alpha} q \geq p\right\}$ and $k(p)(\alpha)=g(q)$ whenever $\alpha \in \operatorname{dom}(k(p))$ and $q$ is the unique element of $A_{\alpha}$ above $p$. It is quite obvious that

(2) $k(p)$ depends only on the set $\left\{q \in D_{0}: q \geq p\right\}$.

\section{Claim 4.}

(3) Whenever $z \in C_{\aleph_{1}}$ is a condition strengthening $k(p)$ then there is $q \leq p$ with $q \Vdash_{P} \check{z} \subset \dot{K}$

(4) $\dot{K}$ is a $P$-name for a $C_{\aleph_{1}}$-generic function and $k$ is the associated projection.

Proof. (3) uses the genericity of the function $g$ over the model $V[f]$. Move into $V[f]$ and let $p \in D, z \in C_{\aleph_{1}}$ and let $x_{0}$ be a condition in the forcing $Q$ adding the function $g$-that is, $x_{0}$ is a finite function from $D$ into 2-such that $x_{0} \Vdash_{Q}$ $\dot{k}(\check{p}) \subset \check{z}$. We shall produce conditions $x_{1} \leq x_{0}$ in $Q$ and $q \leq p$ in $P$ such that $x_{1} \Vdash_{Q} q \Vdash_{P} \check{z} \subset \dot{K}$. By a genericity argument applied to the forcing $Q$ this will complete the proof of $(3)$. By strengthening the condition $x_{0}$ if necessary we 
may assume that $\{s \in D: s \geq p\} \subset \operatorname{dom}\left(x_{0}\right)$, so $x_{0}$ decides the value of $\dot{k}(\check{p})$ to be some $\check{y}, y \in C_{\aleph_{1}}$.

First, by $(\mathrm{C})$ there is a condition $q \leq p$ incompatible with every element of $\operatorname{dom}\left(x_{0}\right)$ which is $¥ p$, and by strengthening $q$ a little more we may assume that for every $\alpha \in \operatorname{dom}(z)$ there is an element $r_{\alpha}$ of $A_{\alpha}$ above $q$. It follows that $\left\{r_{\alpha}: \alpha \in \operatorname{dom}(z)\right\} \cap \operatorname{dom}\left(x_{0}\right)=\left\{r_{\alpha}: \alpha \in \operatorname{dom}(y)\right\}=\left\{r_{\alpha}: \alpha \in \operatorname{dom}(z) \wedge r_{\alpha} \geq p\right\}$ and the set $x_{1}=x_{0} \cup\left\{\left\langle r_{\alpha}, z(\alpha)\right\rangle: \alpha \in \operatorname{dom}(z)\right\}$ is a function, therefore an element of the forcing $Q$ strengthening $x_{0}$ and $x_{1} \Vdash_{Q} q \Vdash_{P} \check{z} \subset \dot{K}$ as desired.

(4) follows from (3) and the fact that $p \Vdash \check{k}(\check{p}) \subset \dot{K}$ by a standard argument.

Now fix an arbitrary isomorphism $\pi: \mathbb{C}_{\aleph_{1}} \rightarrow R O\left(\prod_{\alpha, \beta \in \omega_{1}} B_{\alpha \beta}\right)$. Let $\dot{H}$ be the $P$-name for the image under $\pi$ of the generic filter on $\mathbb{C}_{\aleph_{1}}$ given by the name $\dot{K}$ and let $\dot{E}_{\alpha \beta}$, for limit ordinals $\alpha \in \beta \in \omega_{1}$, be the $P$-names for the closed unbounded subsets of the interval $[\alpha, \beta)$ produced by the $\alpha \beta$-component of the filter $\dot{H}$. Finally, define a $P$-name $\dot{E}$ by $P \Vdash \dot{E}=\bigcup\left\{\dot{E}_{\alpha \beta}: \alpha \in \beta\right.$ are successive elements of the club $\dot{C}$ \}.

We claim that $\dot{E}$ is a $P$-name for a $B$-generic club. In order to show this, first a projection function $h: D \rightarrow B$ will be computed. Fix a condition $p \in D$, choose an element $r_{p} \in \prod_{\alpha, \beta \in \omega_{1}} B_{\alpha \beta}$ with $r_{p} \leq \pi(k(p))$ and write $r_{p}(\alpha, \beta)=$ $\left\langle a_{\alpha \beta}, b_{\alpha \beta}\right\rangle$ whenever the pair $\langle\alpha, \beta\rangle$ is in the finite support of the function $r_{p}$. Set $h(p)=\langle a, b\rangle$ where $a=\operatorname{tr}(p) \cup \bigcup\left\{a_{\alpha \beta}: \alpha \in \beta\right.$ are successive points of $\left.\operatorname{tr}(p)\right\}$ and $b=\bigcup\left\{b_{\alpha \beta}: \alpha \in \beta\right.$ are successive points of $\left.\operatorname{tr}(p)\right\}$.

\section{Claim 5.}

(5) if $p \in D$ and $\langle a, b\rangle \in B$ is a condition strengthening $h(p)$ then there is $q \leq p$ with $q \Vdash \check{a} \subset \dot{E}, \bigcup \check{b} \cap \dot{E}=0$

(6) $\dot{E}$ is a P-name for a B-generic club.

Proof. Towards (5), fix $p \in D$ and a condition $\langle a, b\rangle \in B$ with $\langle a, b\rangle \leq h(p)$. Let $0=\alpha_{0} \in \alpha_{1} \in \cdots \in \alpha_{n}$ be an enumeration of the trace of $p$; for notational convenience set $\omega_{1}=\alpha_{n+1}$. For $m \in n+1$ let $\left\langle a_{m}, b_{m}\right\rangle \in B_{\alpha_{m}, \alpha_{m+1}}$ be the part of the condition $\langle a, b\rangle$ between $\alpha_{m}$ and $\alpha_{m+1}$, that is, $a_{m}=a \cap\left[\alpha_{m}, \alpha_{m+1}\right)$ and $b_{m}=\left\{I \in b: I \subset\left[\alpha_{m}, \alpha_{m+1}\right)\right\}$. Also fix a condition $r=r_{p} \in \prod_{\alpha, \beta \in \omega_{1}} B_{\alpha \beta}$ as in the definition of $h(p)$; by the definitions,

(7) for every $m \in n+1$ we have $\left\langle a_{m}, b_{m}\right\rangle \in B_{\alpha_{m}, \alpha_{m+1}}$ and in that poset it is a stronger condition than $r\left(\alpha_{m}, \alpha_{m+1}\right)$.

For all integers $m \in n+1$ choose an ordinal $\beta_{m}$ strictly between $\alpha_{m}$ and $\alpha_{m+1}$ such that

(8) $\left\langle a_{m}, b_{m}\right\rangle \in B_{\alpha_{m}, \beta_{m}}$ and if $(\chi, \xi) \in \operatorname{supp}(r)$ then $\xi \notin\left[\beta_{m}, \alpha_{m+1}\right)$.

This is easily done since all of the sets $a_{m}, b_{m}, \operatorname{supp}(r)$ are finite while $\alpha_{m+1}$ 's are limits of limit ordinals. 
Subclaim 6. There is a condition $p^{\prime} \leq p$ and ordinals $\gamma_{m}, \beta_{m} \in \gamma_{m} \in \alpha_{m+1}+1$ for all integers $m \in n+1$ such that

(9) $p^{\prime} \Vdash$ "the least element of $\dot{C}$ above $\check{\alpha}_{m}$ is $\check{\gamma}_{m}$, for all $m \in n+1$ "

(10) $\left\{s \in D_{0}: s \geq p^{\prime}\right\}=\left\{s \in D_{0}: s \geq p\right\}$.

Proof. Uses the genericity of the function $f$ over the ground model $V$. Suppose $p \in D, 0=\alpha_{0} \in \beta_{0} \in \alpha_{1} \in \beta_{1} \in \cdots \in \alpha_{n+1}=\omega_{1}$ are as above and $x_{0}$ is a condition in the forcing $R$ adding the function $f$, that is, $x_{0}$ is a finite function from $D$ into 2. We shall produce conditions $x_{1} \leq x_{0}$ in $R$ and $p^{\prime} \leq p$ in $P$ and ordinals $\gamma_{m}: m \in n+1$ such that $p^{\prime}, \gamma_{m}$ satisfy (9) above and $x_{1} \Vdash_{R}\left\{s \in \dot{D}_{0}\right.$ : $\left.s \geq \check{p}^{\prime}\right\}=\left\{s \in \dot{D}_{0}: s \geq \check{p}\right\}$. By a genericity argument with the forcing $R$ this will prove the Subclaim.

Let $u=\left\{s \in \operatorname{dom}\left(x_{0}\right): s \nsupseteq p\right\}$. By the property (C) of the trace function, there is a condition $p^{\prime \prime} \leq p$ which is incompatible with all elements of $u$ and which forces all the sets $\dot{C} \cap\left[\alpha_{m}+1, \beta_{m}\right]$ to be empty, for $m \in n+1$. Choose a strengthening $p^{\prime} \leq p^{\prime \prime}$ deciding in the poset $P$ the values of the minimal elements of the set $\dot{C}$ above $\check{\beta}_{m}$ to be some $\check{\gamma}_{m} \in \check{\alpha}_{m+1}+1$. Obviously the condition $p^{\prime}$ with the ordinals $\gamma_{m}$ satisfy (9) above. It is immediate that $x_{1}=x_{0} \cup\{\langle s, 1\rangle$ : $\left.s \in D, s \geq q, s \notin \operatorname{dom}\left(x_{0}\right)\right\}$ is a function, therefore a condition in the poset $R$ strengthening the condition $x_{0}$ and forcing $\left\{s \in \dot{D}_{0}: s \geq \check{p}\right\}=\left\{s \in \dot{D}_{0}: s \geq\right.$ $\left.\check{p}^{\prime}\right\}=\left\{s \in \operatorname{dom}\left(\check{x}_{0}\right): s \geq \check{p}, \check{x}_{0}(s)=0\right\}$ as desired in (10).

Fix a condition $p^{\prime} \leq p$ and ordinals $\gamma_{m}: m \in n+1$ as in the subclaim and define an element $r^{\prime} \in \prod_{\alpha, \beta \in \omega_{1}} B_{\alpha \beta}$ by setting $\operatorname{supp}\left(r^{\prime}\right)=\left\{\left\langle\alpha_{m}, \gamma_{m}\right\rangle: m \in n+1\right\}$ and $r^{\prime}\left(\alpha_{m}, \gamma_{m}\right)=\left\langle a_{m}, b_{m}\right\rangle$. Now

(11) $r$ and $r^{\prime}$ are compatible elements of the poset $\prod_{\alpha, \beta \in \omega_{1}} B_{\alpha \beta}$.

This holds since if $\gamma_{m}=\alpha_{m+1}$ then $r^{\prime}\left(\alpha_{m}, \gamma_{m}\right)=\left\langle a_{m}, b_{m}\right\rangle \leq r\left(\alpha_{m}, \alpha_{m+1}\right)$ by (7) and if $\gamma_{m} \in \alpha_{m+1}$ then $r^{\prime}\left(\alpha_{m}, \gamma_{m}\right) \leq 1=r\left(\alpha_{m}, \gamma_{m}\right)$ as by (8) the pair $\left\langle\alpha_{m}, \gamma_{m}\right\rangle$ is not included in the support of the condition $r$.

(12) There is a condition $q \leq p^{\prime}$ in $P$ which forces $r^{\prime}$ into the generic filter $\dot{H} \subset \prod_{\alpha, \beta \in \omega_{1}} B_{\alpha \beta}$.

To see this, let $r^{\prime \prime} \in \prod_{\alpha, \beta \in \omega_{1}} B_{\alpha \beta}$ be a common lower bound of the conditions $r, r^{\prime}$. From Subclaim 6(10) and (2) it follows that $k(p)=k\left(p^{\prime}\right)$, and since $r^{\prime \prime} \leq$ $r \leq \pi(k(p))=\pi\left(k\left(p^{\prime}\right)\right)$, Claim 4(3) can be used to find a condition $q \leq p^{\prime}$ which forces $r^{\prime \prime}$ into $\dot{H}$. But $r^{\prime \prime} \leq r^{\prime}$ and so $q$ works for $r^{\prime}$ as well.

By the definitions it now follows that $q \Vdash_{P}$ " $\check{a} \subset \dot{E}, \bigcup \check{b} \cap \dot{E}=0$ " completing the proof of (5). (6) follows by a standard argument.

Stating once again what we have proved in this section, if $D, \dot{C}$ and $t r$ satisfy (A,B,C) then $C_{\aleph_{1}} \Vdash \check{B} \lessdot \check{P}$.

\section{The main forcing}

We shall now describe a proper forcing notion $Q$ which introduces objects $D, \dot{C}, \operatorname{tr}$ into a given nowhere c.c.c. separative $\aleph_{1}$ preserving poset $P$ with uni- 
verse $\omega_{1}$. The poset $Q$ will be closely related to the forcing from [T2, Section 8]. First, some combinatorial notions and related facts which will be instrumental in the proof of properness of $Q$.

Definition 7. [SZ, T2] A set $Y \subset P$ is called small if for every countable subset $\bar{Y} \subset Y$ there is a finite set $u \subset P$ such that every $p \in \bar{Y}$ has some $q \in u$ with $q \leq p$.

Claim 8. Suppose $P$ is a nowhere c.c.c. forcing and $p \in P$. Then the set $\{q \in P: q \leq p\}$ does not belong to the $\sigma$-ideal generated by the small subsets of $P$.

Proof. This is the trivial case of Lemma 21 in [SZ]. Note that small sets cannot contain infinite antichains. Thus if $p \in P$ and $\left\{X_{n}: n \in \omega\right\}$ is a collection of small subsets of the poset $P$, one can choose an uncountable antichain $A \subset P$ consisting of conditions stronger than $p$. Each of the sets $X_{n}: n \in \omega$ can cut out only a finite piece from $A$ and so by a counting argument there must be some $q \in A, q \leq p$ with $q \notin \bigcup_{n} X_{n}$. The Claim follows.

Definition 9. [S] Let $N$ be an arbitrary set. A condition $p \in P$ is called master for $N$ if for every maximal antichain $A \subset P$, an element of $N, p \Vdash$ the one element in the intersection of $\check{A}$ and the generic filter belongs to $\check{N}$. A condition $p \in P$ is called patently not master for $N$ if there is a (maximal) antichain $A \subset P$ in $N$ and a condition $q \in A \backslash N$ with $q \geq p$.

Thus a condition not master for $N$ can be always strengthened into a patently not master condition, and such a condition cannot be strengthened into a master condition any longer.

Claim 10. Suppose $P$ is a nowhere c.c.c. forcing, $\kappa \in \lambda$ sufficiently large cardinals with $H_{\kappa} \in H_{\lambda}$, let $M \prec H_{\lambda}$ be a countable elementary submodel containing $P$ and $\kappa$, let $p \in P$ be a condition and $x \subset M \cap H_{\kappa}$ be a finite set. The there are a countable submodel $N \prec H_{\kappa}$ and a condition $q \leq p$ such that $N \in M, x \subset N$ and $q$ is patently not master for $N$.

Proof. There are two cases depending on whether $p$ is a master condition for $M$ or not. In the former case, note that by the nowhere c.c.c. of the forcing $P$ we have $P \Vdash$ "there are a countable submodel $N \prec \check{H}_{\kappa}$ in the ground model which contains $\check{x}$ and a patently not master condition for $N$ in the generic filter". Since $p$ is a master condition for the model $M$, it forces that such a model $N$ can be found in $M$. So there is a countable model $N \prec H_{\kappa}$ with $N \in M, x \subset N$ and a strengthening $q$ of $p$ which is patently not master for $N$ as desired.

In the latter case, strengthen $p$ into a patently not master condition $q$ for $M$ as witnessed by some maximal antichain $A \subset P$ in $M$. Then $q$ and any countable submodel $N \prec H_{\kappa}$ with $N \in M, x \subset N, A \in N$ witness the statement of the claim.

Finally we are ready to define the proper forcing $Q$. A typical condition in $Q$ is a finite function $f$ with domain a subset in $\omega_{1}$. For $\alpha \in \operatorname{dom}(f)$ the functional 
value $f(\alpha)$ is a quintuple $\left\langle M_{\alpha}^{f}, F_{\alpha}^{f}, p_{\alpha}^{f}, y_{\alpha}^{f}, z_{\alpha}^{f}\right\rangle$ where if no confusion is possible the superscript $f$ is dropped and

(13) $M_{\alpha}$ is an elementary submodel of $H_{\beth_{3}}$ with $\alpha=M_{\alpha} \cap \omega_{1}$ and containing $P$ and $f \uparrow \alpha$ as elements

(14) $F_{\alpha}$ is a finite subset of $H_{\beth_{3}}$

(15) $p_{\alpha} \in P$ is a condition which does not belong to any small subset of $P$ in $M_{\alpha}$

(16) $y_{\alpha} \subset \operatorname{dom}(f) \cap \alpha+1$ and for $\beta \in \operatorname{dom}(f) \cap \alpha+1$ we have $p_{\beta} \geq p_{\alpha} \leftrightarrow \beta \in y_{\alpha}$

(17) $z_{\alpha} \subset \operatorname{dom}(f) \cap \alpha+1$ and for $\beta \in \operatorname{dom}(f) \cap \alpha+1$ we have $p_{\alpha}$ is a master condition for $M_{\beta}$ just in case $\beta \in y_{\alpha}$

(18) there is a condition $q \leq p_{\alpha}$ such that for all $\beta \in \operatorname{dom}(f) \cap \alpha+1 \backslash y_{\alpha}$ the conditions $p_{\beta}$ and $q$ are incompatible and for all $\beta \in \operatorname{dom}(f) \cap \alpha+1 \backslash z_{\alpha}$ the condition $q$ is patently not master for $M_{\beta}$.

The ordering is defined by $f \geq g$ if $\operatorname{dom}(f) \subset \operatorname{dom}(g)$ and for every $\alpha \in$ $\operatorname{dom}(f)$ we have $M_{\alpha}^{f}=M_{\alpha}=g, F_{\alpha}^{f} \subset F_{\alpha}^{g}, p_{\alpha}^{f}=p_{\alpha}^{g}, y_{\alpha}^{f}=y_{\alpha}^{g}$ and $z_{\alpha}^{f}=z_{\alpha}^{g}$.

The definition is long, however the underlying idea is simple. Suppose $G \subset Q$ is a generic filter. The desired set $D \subset P$ will be read off $G$ as $D=\{p \in P$ : $\left.\exists f \in G \exists \alpha \in \operatorname{dom}(f) p=p_{\alpha}^{f}\right\}$. Given a condition $p=p_{\alpha}^{f} \in D$ there are only finitely many elements of $D$ weaker than or equal to it-these are collected in the set $\left\{p_{\beta}: \beta \in y_{\alpha}^{f}\right\}$. To see how the $P$-name $\dot{C}$ for a club is obtained, first define a partial function $\operatorname{Mod}$ on $\omega_{1}$ assigning to $\alpha \in \omega_{1}$ the model $M_{\alpha}^{f}$ where $f \in G$ is an arbitrary condition with $\alpha \in \operatorname{dom}(f)$. It turns out that $\operatorname{dom}(M o d)$ is a closed unbounded subset of $\omega_{1}$ and $M o d$ is a $\subset$-continuous increasing function; therefore the $P$-name $\dot{C}=\{\check{0}\} \cup\left\{\alpha \in \omega_{1}\right.$ : the $P$-generic filter contains a master condition for the model $\operatorname{Mod}(\alpha)\}$ is a name for a club. The trace function will be defined by $\operatorname{tr}\left(p_{\alpha}^{f}\right)=\{0\} \cup z_{\alpha}^{f}$, for any condition $f \in G$ with $\alpha \in \operatorname{dom}(f)$.

Claim 11. The forcing $Q$ is proper.

Proof. Choose a condition $f_{0} \in Q$ and a countable elementary submodel $M \prec$ $H_{\beth_{\omega}}$ containing $Q$ and $f_{0}$ as elements. A master condition $f_{1} \leq f_{0}$ must be produced. First find an element $p \in P$ such that

(19) every $p_{\alpha}^{f_{0}}: \alpha \in \operatorname{dom}\left(f_{0}\right)$ is either greater than or incompatible with $p$ in the poset $P$

(20) $p$ is either master or patently not master for every model $M_{\alpha}^{f_{0}}: \alpha \in$ $\operatorname{dom}\left(f_{0}\right)$ as well as $M$

(21) $p$ does not belong to any of the countably many small subsets of $P$ which happen to be elements of $M$.

It is not hard to see from Claim 8 that there is in fact a dense set in $P$ of such elements-just pick one. Now define the condition $f_{1} \leq f_{0}$ by setting $\alpha=M \cap \omega_{1}$, $\operatorname{dom}\left(f_{1}\right)=\operatorname{dom}\left(f_{0}\right) \cup\{\alpha\}, f_{1} \uparrow \alpha=f_{0}$ and $M_{\alpha}^{f_{1}}=M \cap H_{\beth_{3}}, F_{\alpha}^{f_{1}}=0, p_{\alpha}^{f_{1}}=$ $p, y_{\alpha}^{f_{1}}=\left\{\beta \in \operatorname{dom}\left(f_{1}\right): p_{\beta}^{f_{1}} \geq p\right\}, z_{\alpha}^{f_{1}}=\left\{\beta \in \operatorname{dom}\left(f_{1}\right): p\right.$ is master for the model $\left.M_{\alpha}^{f_{1}}\right\}$.

It is immediate to verify that $f_{1}$ is indeed an element of the forcing $Q$ and 
$f_{1} \leq f_{0}$; item (18) of the definition of $Q$ for $\alpha$ is witnessed by the condition $q=p \leq p=p_{\alpha}^{f_{1}}$. To see that $f_{1}$ is indeed a master condition for the model $M$, suppose $A \in M$ is a maximal antichain of the poset $Q$ and $f_{2} \leq f_{1}$. We must produce a condition $f_{4} \in A \cap M$ compatible with $f_{2}$. By strengthening $f_{2}$ if necessary it can be assumed that $f_{2}$ has an element of $A$ above it.

List the ordinals of $\operatorname{dom}\left(f_{2}\right) \backslash M$ as $M \cap \omega_{1}=\alpha_{0} \in \alpha_{1} \in \cdots \in \alpha_{n}$ and let $f_{3}=f_{2} \uparrow \alpha_{0}$. By item (13) of the definition of the forcing $Q$ we have $f_{3} \in$ $M \cap Q$ and obviously $f_{3} \geq f_{2}$. By induction on $m \in n+2$ we now define certain sets $Z(m): m \in n+2$ whose elements are finite sequences $\eta$ of pairs, $\eta=\left\langle\left\langle N_{0}, p_{0}\right\rangle,\left\langle N_{1}, p_{1}\right\rangle \ldots\right\rangle$ such that $N_{i}$ are countable collections of sets in $H_{\aleph_{2}}$ and $p_{i}$ are elements of $P$. Set

(22) $\eta \in Z(0)$ just in case there is a condition $g \leq f_{3}$ such that $g$ has an element of $A$ above it and $\operatorname{dom}(g)=\operatorname{dom}\left(f_{3}\right) \cup\left\{\beta_{0}, \beta_{1}, \ldots, \beta_{n}\right\}$ for some ordinals $\beta_{i}$ with $\max \left(\operatorname{dom}\left(f_{3}\right)\right) \in \beta_{0} \in \beta_{1} \in \cdots \in \beta_{n}$, and for $i \in n+1$ the $i$-th element of the sequence $\eta$ is $\left\langle M_{\beta_{i}}^{g} \cap H_{\aleph_{2}}, p_{\beta_{i}}^{g}\right\rangle$

(23) $\eta \in Z(m+1)$ if for every finite set $x \subset H_{\aleph_{2}}$ the set $\{p \in P$ : there is some $N$ with $x \subset N$ and $\left.\eta^{\frown}\langle N, p\rangle \in Z(m)\right\}$ is not small in the partial order $P$.

Note that the collection $Z(m): m \in n+1$ belongs to $H_{\beth_{3}} \cap M=M_{\alpha_{0}}^{f_{2}}$ and with it to all of the models $M_{\alpha_{i}}^{f_{2}}: i \in n+1$. Now

(24) $0 \in Z(n+1)$.

For suppose not. Using the elementarity of the models $M_{\alpha_{m}}^{f_{2}}$ and requirement (15) of the definition of the forcing $Q$ it is then possible to prove by induction on $m \in n+2$ that the sequence $\eta_{m}=\left\langle\left\langle M_{\alpha_{0}}^{f_{2}} \cap H_{\aleph_{2}}, p_{\alpha_{0}}^{f_{2}}\right\rangle,\left\langle M_{\alpha_{1}}^{f_{2}} \cap\right.\right.$ $\left.\left.H_{\aleph_{2}}, p_{\alpha_{1}}^{f_{2}}\right\rangle, \ldots\left\langle M_{\alpha_{m-1}}^{f_{2}} \cap H_{\aleph_{2}}, p_{\alpha_{m-1}}^{f_{2}}\right\rangle\right\rangle$ is not in $Z(n+1-m)$. However, by the definition of $Z(0)$, the sequence $\eta_{n+1}$ is in $Z(0)$ as witnessed by the condition $f_{2} \leq f_{3}$, a contradiction.

Choose conditions $q^{m} \leq p_{\alpha_{m}}^{f_{2}}: m \in n+1$ in the poset $P$ which witness (18) of the definition of $Q$ for $f_{2}$ and $\alpha_{m}: m \in n+1$. By induction on $i \in n+1$ build pairs $\left\langle N_{i}, p_{i}\right\rangle$ and conditions $q_{i}^{m}: m \in n+1$ in the poset $P$ so that

(25) $N_{i}, p_{i} \in M$ and the sequence $\eta_{i}=\left\langle\left\langle N_{0}, p_{0}\right\rangle,\left\langle N_{1}, p_{1}\right\rangle \ldots\left\langle N_{i-1}, p_{i-1}\right\rangle\right\rangle$ belongs to $Z(i)$

(26) $q^{m} \geq q_{0}^{m} \geq q_{1}^{m} \geq \ldots$ for all $m \in n+1$

(27) $p_{i}$ is a condition in $P$ incompatible with all $q_{i}^{m}: m \in n+1$

(28) $N_{i}$ is a countable submodel of $H_{\aleph_{2}}$ for which all of the conditions $q_{i}^{m}$ : $m \in n+1$ are patently not master.

Once this is achieved by the elementarity of the model $M$ and the definition of the set $Z(0) \in M$ there is a condition $g \in M \cap Q$ witnessing that $\eta_{n+1} \in Z(0)$. We claim that $f_{2}, g$ are compatible elements of $Q$ with a common lower bound $h=f_{2} \cup g$. It only has to be verified that $h \in Q$ and there again the only nontrivial point to see is why (18) holds for $h$ and the ordinals $\alpha_{m} \in \operatorname{dom}\left(f_{2}\right) \subset$ $\operatorname{dom}(h): m \in n+1$. But obviously the condition $q_{n}^{m} \leq p_{\alpha_{m}}^{f_{2}}=p_{\alpha_{m}}^{h}$ has been constructed so as to witness (18) for $h$ and $\alpha_{m}$. Now $f_{2}$ has an element of $A$ 
above it by its choice and $g$ has an element of $A$ above it by the definition of the set $Z(0)$. Since $f_{2}, g$ are compatible conditions in $Q$, these two members of the antichain $A$ must be identical, equal to some $f_{4} \in A$. By the elementarity of the model $M$, since $f_{4}$ is the only element of $A \in M$ above $g \in M$ it must be the case that $f_{4} \in M$. Since $f_{4} \in A \cap M$ and $f_{4} \geq f_{2}$, the properness of the forcing $Q$ follows.

Now suppose $\left\langle N_{i}, p_{i}\right\rangle$ and $q_{i}^{m}: m \in n+1$ have been constructed for all $i \in j$ for some integer $j \in n$. To obtain the pair $\left\langle N_{j}, p_{j}\right\rangle$ and the conditions $q_{j}^{m}: m \in n+1$ first by induction on $k \in n+1$ build models $X_{k}$, maximal antichains $A_{k} \subset P$ and conditions $r^{k} \leq q_{j-1}^{k}$ (or $r^{k} \leq q^{k}$ if $j=0$ ) so that

(29) $X_{k}$ is a countable elementary submodel of $H_{\beth_{n+4-k}}$ containing the poset $P$ and the collection $Z(m): m \in n+2$ and $M \ni X_{0} \ni X_{1} \ni \ldots$

(30) $\left\{A_{l}: l \in k+1\right\} \subset X_{k}$ for all $k \in n+1$

(31) $r^{k}$ is a condition in $P$ patently not master for the model $X_{k}$ as witnessed by the maximal antichain $A_{k}$, that is, there is $s \in A_{k} \backslash X_{k}$ with $s \geq r^{k}$.

This is rather easily done using Claim 10 at each stage $k$ of the induction to the model $X_{k-1}$ (or $M$ if $k=0$ ), the condition $q_{j-1}^{k} \in P$ and the finite set $\left\{A_{l}\right.$ : $l \in k, Z(m): m \in n+1\} \subset X_{k-1}$. Note that in the end, $\left\{A_{k}: k \in n+1\right\} \subset X_{n}$ and each of the conditions $r^{k} \leq q_{j-1}^{k}: k \in n+1$ is patently not master for the model $X_{n} \prec H_{\beth_{4}}$ as witnessed by the maximal antichain $A_{k} \subset P$.

Since the sequence $\eta_{j}$ belongs to the set $Z(n+1-j)$ by the induction hypothesis-or (24) if $j=0$-, it follows that the set $Y=\left\{p \in P: \exists N\left\{A_{k}\right.\right.$ : $\left.k \in n+1\} \subset N \wedge \eta_{j}\langle N, p\rangle \in Z(n-j)\right\} \subset P$ is not small. By the definition of smallness there is a countable set $\bar{Y} \subset Y$ such that for every finite set $u \subset P$ there is $p \in \bar{Y}$ such that for all $s \in u s \not \leq p$ holds. Since $Y \in X_{n}$, by the elementarity of the model $X_{n}$ we can find such a set $\bar{Y} \subset Y$ as an element and therefore subset of $X_{n}$. By the choice of $\bar{Y}$ there is an element $p_{j} \in \bar{Y}$ such that for all $m \in n+1 r^{m} \not \leq p_{j}$ holds and one can choose conditions $q_{j}^{m} \leq r^{m}: m \in n+1$ which are incompatible with $p_{j}$ by the separativity of the poset $P$. Finally, since $p_{j} \in \bar{Y} \subset X_{n} \cap Y$ it is possible by the definition of the set $Y$ and the elementarity of the model $X_{n}$ to choose a countable set $N_{j} \subset H_{\aleph_{2}}$ such that $\left\{A_{m}: m \in n+1\right\} \subset N_{j} \in X_{n}$ and $\eta_{j+1}=\eta_{j}\left\langle N_{j}, p_{j}\right\rangle \in Z(n-j)$.

We claim that the induction hypotheses continue to hold with the pair $\left\langle N_{j}, p_{j}\right\rangle$ and conditions $q_{j}^{m}: m \in n+1$. And indeed, the hypotheses $(25,26,27)$ were explicitely arranged to hold in the previous paragraph. To see why (28) holds, fix $m \in n+1$ and observe that $A_{m} \in N_{j} \subset X_{n+1} \subset X_{m+1}$. Now the induction construction performed in (29-31) provides an element $s \in A_{m} \backslash X_{m+1} \subset A_{m} \backslash N_{j}$ such that $q_{j}^{m} \leq r^{m} \leq s$. Thus the condition $q_{j}^{m}$ is patently not master for the model $N_{j}$ as witnessed by the maximal antichain $A_{m} \in N_{j}$. This concludes the inductive construction in (25-28) and the proof of the properness of the forcing $Q$.

All that remains to be done is some density arguments proving that the forcing $Q$ adjoins the desired objects to the universe. Suppose $G \subset Q$ is a generic filter 
and work in $V[G]$. Let $D=\left\{p_{\alpha}^{f} \in P: f \in G, \alpha \in \operatorname{dom}(f)\right\}$.

Claim 12. $D \subset P$ is a dense set and for every $p \in D$ the set $\{q \in D: q \geq p\}$ is finite.

Proof. Back in $V$, suppose that $p \in P$ and $f \in Q$ are conditions. We will produce strengthenings $q \leq p$ in $P$ and $g \leq f$ in $Q$ such that $g \Vdash_{Q}$ " $\check{q} \in \dot{D}$ " which by a genericity argument applied with the forcing $Q$ proves the density of the set $D \subset P$. Choose a countable elementary submodel $M \prec H_{\beth_{3}}$ with $P, f \in M$. As in the first paragraph of the proof of the properness of $Q$ the set $Y=\{q \in P$ :there are finite sets $y, z$ such that $\left.g=f \cup\left\{\left\langle M \cap \omega_{1},\langle M, 0, q, y, z\rangle\right\rangle\right\} \in Q\right\} \subset P$ is dense. Pick some $q \leq p$ in $Y$ and $g \in Q$ witnessing that $q \in Y$; then $g \leq f$ and

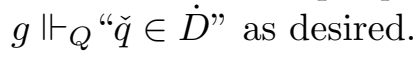

For the finite character of the set $D$ note that if $f \in Q$ and $\alpha \in \operatorname{dom}(f)$ then $f \Vdash_{Q} "\left\{q \in \dot{D}: q \geq \check{p}_{\alpha}^{f}\right\}=\left\{p_{\beta}^{f}: \beta \in \check{y}_{\alpha}^{f}\right\} "$ by the definitions.

Define a partial function $\operatorname{Mod}$ with $\operatorname{dom}(\operatorname{Mod}) \subset \omega_{1}$ by $\operatorname{Mod}(\alpha)=M_{\alpha}^{f}$ if there is a condition $f \in G$ with $\alpha \in \operatorname{dom}(f)$ and $\operatorname{Mod}(\alpha)$ is left undefined otherwise. Let $\dot{C}$ be the $P$-name given by $P \Vdash \dot{C}=\{\check{0}\} \cup\{\alpha \in \operatorname{dom}(\operatorname{Mod})$ : there is a master condition in the $P$-generic filter for the model $\operatorname{Mod}(\alpha)\}$.

\section{Claim 13.}

(32) $\operatorname{dom}(M o d) \subset \omega_{1}$ is a closed unbounded set

(33) Mod is a $\subset$-continuous increasing function

(34) $P \Vdash \dot{C} \subset \check{\omega}_{1}$ is a closed unbounded set.

Proof. Move back to $V$ to prove (32) and (33). For the closedness of the set $\operatorname{dom}(M o d)$ suppose $f \in Q$ and $f \Vdash \check{\beta} \in \check{\omega}_{1}$ is a limit point of the set $\operatorname{dom}(\operatorname{Mod})$. We shall show that $f \Vdash " \check{\beta} \in \operatorname{dom}(M o d)$ ", which is certainly enough. Suppose this fails and pick a strengthening $g \leq f$ with $g \Vdash \check{\beta} \notin \operatorname{dom}(M o d)$. Without loss of generality we may assume that $\operatorname{dom}(g) \cap \beta \neq 0$ and let $\alpha=\max (\beta \cap \operatorname{dom}(g))$. Define a condition $h \leq g$ by copying $g$ with the only change of setting $F_{\alpha}^{h}=F_{\alpha}^{g} \cup$ $\{\beta\}$. By the definition of the forcing $Q$-item $(13)-h \Vdash \check{\alpha}=\max (\operatorname{dom}(M o d)) \cap \check{\beta}$ and so $\check{\beta}$ is not a limit point of the set $\operatorname{dom}(\operatorname{Mod})$, a contradiction.

For the unboundedness of $\operatorname{dom}(M o d)$ fix a condition $f \in Q$ and an ordinal $\alpha \in \omega_{1}$. Choose a countable elementary submodel $M \prec H_{\beth_{3}}$ with $P, f, \alpha \in M$ and as in the proof of properness of $Q$ find a condition $p \in P$ and finite sets $y, z$ such that $h=g \cup\left\{\left\langle M \cap \omega_{1},\langle M, 0, p, y, z\rangle\right\rangle\right\} \in Q$. Obviously $h \leq f$ and $h \Vdash \check{\alpha} \in \check{M} \cap \omega_{1} \in \operatorname{dom}(M o d)$. The unboundedness of $\operatorname{dom}(M o d)$ in $\omega_{1}$ now follows by a genericity argument.

(33) is proved in much the same way as (32). Note that the function Mod is $\subset$-increasing due to the requirement (13) in the definition of $Q$.

To see that $P \Vdash$ " $\dot{C}$ is an unbounded subset of $\check{\omega}_{1}$ " fix conditions $f \in Q$ and $p \in P$ and an ordinal $\alpha \in \omega_{1}$. We shall produce strengthenings $g \leq f$ in $Q$ and $q \leq p$ in $P$ and an ordinal $\beta>\alpha$ such that $g \Vdash_{Q} q \Vdash_{P} \check{\beta} \in \dot{C}$. By a genericity argument used with $Q \times P$ this will prove the unboundedness of $\dot{C}$ as forced by 
$P$ in $V[G]$. Here is the only place where the assumption of $P$ preserving $\aleph_{1}$ is used. It makes it possible to choose a countable elementary submodel $M \prec H_{\beth_{3}}$ with $\alpha, p, f, P \in M$ and a master condition $q \leq p$ in $P$ for $M$. As in the first paragraph of the proof of properness there are a condition $r \in P$ and finite sets $y, z$ such that $g=f \cup\left\{\left\langle M \cap \omega_{1},\langle M, 0, r, y, z\rangle\right\rangle\right\}$ belongs to $Q$. Obviously $g \leq f$ and $g \Vdash_{Q} q \Vdash_{P} \check{\beta}=\check{M} \cap \check{\omega}_{1} \in \dot{C}$ as desired.

The fact that $P \Vdash$ " $\dot{C}$ is closed below $\check{\omega}_{1}$ " can be proved in $V[G]$ by an appeal to (33) above. Work in $V[G]$ and suppose $p \in P$ and $\beta \in \omega_{1}$ are such that $p \Vdash \check{\beta}$ is a limit point of $\dot{C}$. It will be shown that $p$ is a master condition for $\operatorname{Mod}(\beta)$ meaning that $p \Vdash " \check{\beta} \in \dot{C}$ " and implying the forced closedness of the set $\dot{C}$. And indeed, if $p$ were not a master condition for $\operatorname{Mod}(\beta)$ one could find a strengthening $q \leq p$ patently not master for $\operatorname{Mod}(\beta)$ and and an antichain $A \in \operatorname{Mod}(\beta)$ witnessing it. By (33) above there is $\alpha \in \beta$ such that $A \in \operatorname{Mod}(\alpha)$. Obviously, the condition $q$ is patently not master for all of the models $\operatorname{Mod}(\gamma): \alpha \in \gamma \in \beta$ as witnessed by $A$ and so $q \Vdash \dot{C} \cap \check{\beta}$ is bounded by $\check{\alpha}$ and $\check{\beta}$ is not a limit point of $\dot{C}$, a contradiction.

Finally we must define the trace function and verify the point $(\mathrm{C})$ from the previous section. For $p \in D, p=p_{\alpha}^{f}$ for some $f \in G$ and $\alpha \in \operatorname{dom}(f)$ let $\operatorname{tr}(p)=\{0\} \cup z_{\alpha}^{f}$. By the definitions and (17), $p \Vdash_{P} \operatorname{tr}(p) \subset \dot{C}$.

Claim 14. Suppose $p \in D, u \subset D$ is a finite set of conditions $¥ p$ and $b$ is $a$ finite set of clopen bounded intervals of countable ordinals disjoint from $\operatorname{tr}(p)$. Then there is a strengthening $q \leq p$ which is incompatible with every element of $u$ and forces in $P$ that $\bigcup \check{b} \cap \dot{C}=0$.

Proof. Move back to $V$ and pick $f \in Q, p \in P, u \subset P$ and a finite set $b$ of ordinal intervals such that $f \Vdash_{Q} \check{p}, \check{u}, \check{b}$ are as in the claim. By strengthening the condition $f$ if necessary we may assume that for each $s \in u \cup\{p\}$ there is some $\alpha \in \operatorname{dom}(f)$ with $s=p_{\alpha}^{f}$ and that $f \Vdash \min (\operatorname{dom}(M o d))=\min (\operatorname{dom}(f))$. We shall produce conditions $q \leq p$ in $P$ and $g \leq f$ in $Q$ such that $g \Vdash_{Q} \check{q}$ is as in the conclusion of the claim. By a genericity argument applied with the forcing $Q$ this will certainly complete the proof.

Fix an ordinal $\alpha \in \operatorname{dom}(f)$ such that $p=p_{\alpha}^{f}$; note that $f \Vdash_{Q}$ " $\operatorname{tr}(\check{p})=\{0\} \cup$ $\left\{z_{\alpha}^{f}\right\}$ " and $u \cap\left\{p_{\beta}^{f}: \beta \in y_{\alpha}^{f}\right\}=0$ by $(16,17)$ of the definition of the forcing $Q$. The superscript $f$ will be left out in expressions throughout the rest of the proof. A strengthening $q \leq p$ will be constructed so that for each $\beta \in \operatorname{dom}(f) \backslash y_{\alpha}$ the conditions $p_{\beta}$ and $q$ are incompatible and for every $\beta \in \operatorname{dom}(f) \backslash z_{\alpha}$ the condition $q$ is patently not master for the model $M_{\beta}$. Suppose this has been done. Then define a function $g \leq f$ in $Q$ by copying $f$ with only the following changes:

(35) Suppose $\beta \in \operatorname{dom}(f)$ is an ordinal such that $\gamma=\min (\operatorname{dom}(f) \backslash \beta+1)$ exists and $q$ is patently not master for the model $M_{\gamma}$ as witnessed by an antichain $A \subset P$. Then let $F_{\beta}^{g}=F_{\beta}^{f} \cup\{A\}$. This implies that $g \Vdash_{Q}$ "every $\operatorname{model} \operatorname{Mod}(\xi): \beta \in \xi \in \gamma+1$ contains the antichain $\check{A}$, so $\check{q}$ is patently not master for it, so $q \Vdash_{P} \dot{C} \cap(\beta, \gamma]=0$ ". 
(36) Suppose $\beta \in \operatorname{dom}(f)$ is an ordinal such that $\gamma=\min (\operatorname{dom}(f) \backslash \beta+1)$ exists and $q$ is a master condition for the model $M_{\gamma}$-so $\gamma \in z_{\alpha}$. Then since $b$ is a finite set of clopen intervals of ordinals which do not contain $\gamma$, certainly $\delta=\max (\bigcup b \cap \gamma)$ exists and is smaller than $\gamma$. Let $F_{\beta}^{g}=$ $F_{\beta}^{f} \cup\{\delta\}$. This is to make certain that $g \Vdash_{Q} \cdot \operatorname{dom}(M o d) \cap(\check{\beta}, \check{\delta}]=0$ and so $q \Vdash_{P} \dot{C} \cap \bigcup b \cap(\beta, \gamma)=0 "$.

(37) $\beta$ is the maximal element of $\operatorname{dom}(f)$. Then let $\delta=\max \bigcup b$ and $F_{\beta}^{g}=$ $F_{\beta}^{f} \cup\{\delta\}$. This implies that $g \Vdash_{Q} \cdot \operatorname{dom}(M o d) \cap(\check{\beta}, \check{\delta}]=0$, so $q \Vdash_{P}$ $\dot{C} \cap \bigcup b \backslash \beta+1=0 "$.

All seen and told, the condition $q$ is incompatible with every element of $u$ and $g \Vdash_{Q} q \Vdash_{P} \dot{C} \cap \bigcup b=0$ as desired.

Now onto the construction of the condition $q \leq p$ in $P$. Let $\alpha \in \alpha_{0} \in \alpha_{1} \in$ $\cdots \in \alpha_{n}$ be an enumeration of the ordinals in $\operatorname{dom}(f)$ above $\alpha$ and by induction construct a chain $p \geq q_{0} \geq q_{1} \geq \cdots \geq q_{n+1}$ in $P$ so that

(38) if $i \in n$ then $q_{i} \in M_{\alpha_{i}}$

(39) $q_{0}$ witnesses item (18) of the definition of $Q$ for $f$ and $\alpha$

(40) $q_{i+1}$ is patently not master for $M_{\alpha_{i}}$ and is incompatible with the condition $p_{\alpha_{i}}$, for all $i \in n+1$.

In the end, $q=q_{n+1}$ will be as desired. Now obviously $q_{0} \in M_{\alpha_{0}}$ can be chosen as desired by (18) and the elementarity of the model $M_{\alpha_{0}}$. Suppose $q_{i} \in M_{\alpha_{i}}$ has been constructed for some $i \in n+1$. Then choose an uncountable antichain $A \subset P$ in the model $M_{\alpha_{i}}$ consisting of conditions stronger than $q_{i}$. There must be an element $s \in A \backslash M_{\alpha_{i}}$ which is not stronger than $p_{\alpha_{i}}$; otherwise $p_{\alpha_{i}} \in\{r \in P$ : for all but countably many $s \in A s \leq r$ holds $\}$, the latter set is small and a member of $M_{\alpha_{i}}$ and a contradiction with (15) results. So choose such $s \in A \backslash M_{\alpha_{i}}$ and a strengthening $q_{i+1} \leq s \leq q_{i}$ which is incompatible with the condition $p_{\alpha_{i}}$ using the separativity of the forcing $P$. Obviously $q_{i+1}$ satisfies (40) above; if $i \in n$ then the whole construction can be performed in $M_{\alpha_{i+1}}$ to obtain $q_{i+1} \in M_{\alpha_{i+1}}$ as in (38). This concludes the induction and the proof of the Claim.

The previous three claims show that the proper forcing $Q$ adds objects $D, \dot{C}, t r$ with the properties $(\mathrm{A}, \mathrm{B}, \mathrm{C})$ from the previous section, and so by the results of that section it follows that $Q \times C_{\aleph_{1}} \Vdash \check{B} \lessdot \check{P}$.

Theorem 2 is now a routine application of the Proper Forcing Axiom. Suppose $P$ is a nowhere c.c.c. poset of size $\aleph_{1}$, and write it as a disjoint union $P_{0} \cup P_{1}$ where $P_{0}$ collapses $\aleph_{1}$ outright while $P_{1}$ preserves it. By [J, Lemma 25.11] $R O\left(P_{0}\right)$ is isomorphic to $\mathbb{D}$ and so certainly it contains a complete copy of $\mathbb{B}$; thus without loss of generality we may assume that $P=P_{1}, P$ preserves $\aleph_{1}$ and its universe is $\omega_{1}$. By the results of this and the previous section there is a proper forcing $Q$ such that $Q \times C_{\aleph_{1}} \Vdash \check{B} \lessdot \check{P}$. Note that $Q \times C_{\aleph_{1}}$ is a proper forcing notion as well and so by standard PFA considerations [SZ, Lemma 38] there are a cardinal $\kappa$, an elementary submodel $M \prec H_{\kappa}$ and a filter $G \subset M \cap Q \times C_{\aleph_{1}}$ 
such that $\omega_{1} \subset M, P, Q \in M$ and $G$ meets every dense subset of $Q \times C_{\aleph_{1}}$ which happens to be an element of the model $M$. Let us write ${ }^{-}: M \rightarrow \bar{M}$ for the transitive collapse map and $\bar{G}$ for the image of the filter $G$ under this map. Note $\bar{P}=P$ and $\bar{B}=B$ since $\omega_{1} \subset M$. So by the elementarity of the model $M$ we have $\bar{M}[\bar{G}] \models B=\bar{B} \lessdot \bar{P}=P$. Now it is a standard fact of the forcing theory that $B \lessdot P$ is a $\Sigma_{1}$ statement about the orderings $B$ and $P$ : it is equivalent to an existence of a poset $R$ on $B \cup P$ extending the respective orderings and comparability and compatibility relations such that $P$ is dense in $R$ and $B$ is regular in $R(\forall r \in R \exists b \in B \forall c \in B c \leq b \rightarrow c$ and $r$ are compatible in $R)$. Thus $\bar{M}[\bar{G}] \models B \lessdot P$ implies that there is such an ordering $R \in \bar{M}[\bar{G}]$; it keeps its properties even if viewed from the universe $V$ and therefore $B \lessdot P$ holds. Theorem 2 follows.

Theorem 3 is proved by repeating all of the above arguments literally just replacing the forcings $B$ and $B_{\alpha \beta}$ with $B_{\omega_{1} \backslash S}$ and $B_{[\alpha, \beta) \backslash S}$. Note that a poset collapsing the stationarity of a set $S \subset \omega_{1}$ is automatically nowhere c.c.c.

If one wishes to obtain the equiconsistency of the relationships from the figure in the introduction with ZFC, it is necessary to upgrade the forcing $Q$ of this section with matrices of models as side conditions $[\mathrm{SZ}, \mathrm{T}]$ in order to obtain an $\omega_{2}$-p.i.c. [S] notion of forcing which then can be iterated $\omega_{2}$ many times without collapsing cardinals. Since this is a rather standard and notationally complex procedure, we opt to leave it out.

\section{References}

[B] J. E. Baumgartner, Applications of the proper forcing axiom, Handbook of set-theoretical topology (K. Kunen and J. E. Vaughan, eds.), North Holland, Amsterdam, 1984, pp. 913959.

[J] T. Jech, Set Theory, Academic Press, New York, 1978.

[K] S. Koppelberg, Characterization of Cohen algebras, Papers on general topology and applications (S. Andima, R. Kopperman, P. R. Misra and A. R. Todd, eds.), Annals of The New York Academy of Sciences 704, New York, 1993, pp. 227-237.

[S] S. Shelah, Proper Forcing, Lecture Notes in Mathematics 940, Springer-Verlag, Berlin, 1981.

[SZ] S. Shelah and J. Zapletal, Embeddings of Cohen algebras, Adv. Math. 126 (1997), 93-119.

[T1] S. Todorcevic, A note on the proper forcing axiom, Axiomatic Set Theory (J. E. Baumgartner, D.A. Martin and S. Shelah, eds.), Contemp. Math. 31, Amer. Math. Soc., Providence, 1984.

[T2] , A classification of transitive relations on $\omega_{1}$, Proc. London Math. Soc. 73 (1996), $501-533$.

[Z1] J. Zapletal, Characterization of the club forcing, Papers on general topology and applications (S. Andima, R. C. Flagg, G. Itzkowitz, Y. Kong, R. Kopperman, P. Misra, eds.), Annals of The New York Academy of Sciences 806, New York, 1996, pp. 476-484.

[Z2] _ A classification of definable partial orders on $\omega_{1}$, Fund. Math. (to appear).

Mail Code 253-37, California Institute of Technology, Pasadena, CA 91125

E-mail address: jindra@cco.caltech.edu 